\title{
Bleomycin sclerotherapy for large diffuse microcystic lymphatic malformations
}

\author{
Lingling Sheng, Ziyou Yu, Shengli Li, Weigang Cao, Zhaohua Jiang \\ Department of Plastic and Reconstructive Surgery, Shanghai Ninth People's Hospital, School of Medicine, Shanghai Jiao Tong University, Shanghai, \\ China \\ Contributions: (I) Conception and design: Z Jiang; (II) Administrative support: S Li, W Cao; (III) Provision of study materials or patients: L Sheng, Z \\ Yu; (IV) Collection and assembly of data: L Sheng, Z Yu; (V) Data analysis and interpretation: L Sheng, Z Jiang; (VI) Manuscript writing: All authors; \\ (VII) Final approval of manuscript: All authors. \\ Correspondence to: Zhaohua Jiang, MD, PhD. Department of Plastic and Reconstructive Surgery, Shanghai Ninth People's Hospital, School of \\ Medicine, Shanghai Jiao Tong University, 639 Zhi Zao Ju Road, Shanghai 200011, China. Email: zhhjiang@163.com.
}

\begin{abstract}
Background Microcystic lymphatic malformations (LMs) are congenital lesions with the diameter of the majority of cysts $<1 \mathrm{~cm}$. Bleomycin sclerotherapy has been shown to yield beneficial results for macrocystic LMs. This study aims to evaluate the safety and efficacy of consecutive bleomycin sclerotherapy for large diffuse microcystic LMs.

Methods: The location and size of the lesions were detected by ultrasound for the 46 patients included in this study. Bleomycin lavage was performed in larger cysts and intradermal injection for the superficial lesion. The outcome and complications were assessed for its efficacy and safety.

Results: The large diffuse microcystic LMs mainly located in the neck, abdominal wall and axilla/lateral chest wall. The average lesion size was $10.6 \mathrm{~cm} \times 7.2 \mathrm{~cm}$. The mean number of treatment sessions was 4.5 with $7.3 \mathrm{mg}$ bleomycin for per session averagely. Excellent (69.6\%) and moderate (23.9\%) responses were obtained. There was no recurrence for the 6 patients (13\%) who received a long follow-up. Obvious local swelling, slight intralesional hemorrhage and low-grade fever were the most commonly occurred complications. No lung fibrosis was identified for the patients who received more than 6 sessions.

Conclusions: Local lavage combined with intradermal injection of bleomycin is effective and safe for large diffuse microcystic LMs with good therapeutic effect and low complication rates, and can be regarded as the mainstay of therapy for microcystic LMs.
\end{abstract}

Keywords: Pulmonary fibrosis; microcystic lymphatic malformations (microcystic LMs); bleomycin injection; sclerotherapy

Submitted Feb 02, 2021. Accepted for publication May 07, 2021.

doi: $10.21037 / g s-21-70$

View this article at: https://dx.doi.org/10.21037/gs-21-70

\section{Introduction}

Lymphatic malformations (LMs) are congenital anomalies due to disorder of vascular embryogenesis, and consist of abnormally formed lymphatic channels and cystic spaces of varying size (1). LMs are more commonly seen in lymphatic-rich areas, such as the head and neck (45-52\%), axilla, mediastinum, groin, and retroperitoneum (2). Histologically, the cysts are lined with a single layer of lymphatic endothelial cells and abnormal smooth and skeletal muscular structures, and filled with eosinophilic, protein-rich fluid (3). LMs can be solitary or multifocal, and are classified as macrocystic $(>1 \mathrm{~cm})$, microcystic $(<1 \mathrm{~cm})$, or mixed based on the diameter of the majority of cysts in the lesion (4). Microcystic LMs often present as clusters of clear, tiny vesicles that permeate the subcutaneous tissue and muscles, and show a brawny edema.

There are various treatments for LMs, including surgical excision, radiation therapy, cryotherapy, electrocautery, sclerotherapy, steroid administration, embolization and ligation, laser surgery, radio-frequency tissue ablation 


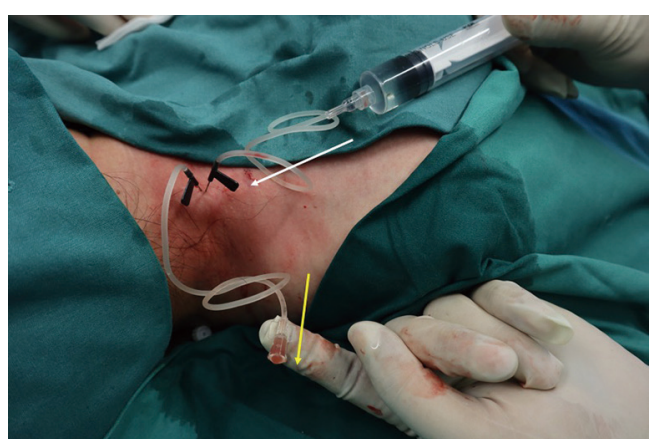

Figure 1 Schematic diagram of bleomycin lavage. The two scalp punctures were inserted into the cyst with a distance of 2-3 $\mathrm{mm}$. After observing the lymphatic fluid reflux, bleomycin was injected from one tube (white arrow), and the overflowing lymphatic fluid and bleomycin will flow out from the other tube (yellow arrow).

technique, and combinations of these treatments (5-8). Macrocystic LMs respond well to sclerotherapy and surgical resection $(9,10)$. For microcystic LMs, there is no consensus on the optimal treatment. Surgical resection used to be the main treatment of microcystic LMs $(11,12)$, but radical excision of the lesions is often impossible because of the diffuse extent and inaccurate determination of the lesion boundary, leading to a high recurrence of $35-100 \%$ (13). In addition, as a surgery, it inherently may cause postoperative complications, such as lymphatic fluid leakage, nerve damage, hematoma formation, and hypertrophic scars. These shortcomings make surgical treatment a less desirable option for microcystic LMs.

In recent years, encouraging results have been obtained in bleomycin sclerotherapy for microcystic LMs $(14,15)$. Bleomycin plays a sclerosing effect on the endothelial cells with nonspecific inflammatory reaction to occlude the malformed lymphatic vessels (16). However, its effect and safety on large diffuse microcystic LMs are still unclear. The purpose of this study is to evaluate the efficacy and safety of sclerotherapy with bleomycin based on pre- and post-procedural clinical data and magnetic resonance imaging (MRI) examination.

We present the following article in accordance with the STROBE reporting checklist (available at https://dx.doi. org/10.21037/gs-21-70).

\section{Methods}

All procedures were performed in accordance with the ethical standards of the institutional review board (approval from Comissão de Ética do Hospital dos SAMS, 002/2018) and with the 1964 Helsinki declaration and its later amendments (as revised in 2013) or comparable ethical standards. This study was approved by the research and ethics committee of the Shanghai Ninth People's Hospital (registration number: 2019042). Written informed consent was obtained from all patients.

This retrospective study included 46 patients who were diagnosed as large diffused microcystic LMs ( $>1 \%$ body surface area) between January 2012 and December 2019 according to the medical history, imaging, and clinical manifestations. The following parameters were noted, including patient age, sex, location and size of the lesion, previous treatments, sessions of procedures, dose of bleomycin, complications, clinical and imaging follow-up. Before treatment, all the patients were detected with chest $\mathrm{X}$-ray plain film and a routine blood test.

\section{Sclerotherapy}

Preoperative ultrasound examinations combined with MRI or magnetic resonance lymphangiography (MRL) were used to confirm the diagnosis and provide accurate information regarding the size and location of the cysts, the extent of the abnormality and connection with surrounding tissue. Bleomycin powder $(10 \mathrm{mg})$ was reconstituted with $10 \mathrm{~mL}$ $0.9 \%$ saline to give a final concentration of $1 \mathrm{mg} / \mathrm{mL}$.

The entry points received local anesthesia with $2 \%$ lidocaine. According to the preoperative location by ultrasound and palpation, two $22 \mathrm{G}$ scalp acupunctures were punctured into the large cysts whose diameter was $>5 \mathrm{~mm}$ with a distance of $2-3 \mathrm{~mm}$, and the lymphatic fluid reflux into the tubes was always observed. It should be taken attention not to puncture into vessels to minimize unwanted bleomycin intake. The overflowed lymphatic fluid was aspirated to optimize the effect. To minimize lung toxicity, intralesional bleomycin lavage was performed between the two scalp acupunctures at a concentration of $1 \mathrm{mg} / \mathrm{mL}$ at a flow rate of $1 \mathrm{~mL} / \mathrm{min}$ in the larger cysts (Figure 1). Four to six intralesional lavages points were performed into the larger components of the microcystic LMs. For tiny cysts in dermal tissue, bleomycin was injected intradermally with hypodermic needles for even dispersion. The sclerotherapy was repeated at 4- to 6-week intervals at the dose of 5-10 mg each time, not exceeding $10 \mathrm{mg}$. To promote adhesion of cysts and reduce drug extravasation, pressure dressing after treatment was used for 24 hours. All the patients were observed for 24 hours in hospital in case of adverse events. 
Table 1 Characteristics of the study subjects

\begin{tabular}{lc}
\hline Characteristics & $\mathrm{N}=46$ \\
\hline Age, years & $23 \pm 4$ (range, 6-37) \\
Sex & 25 \\
Male & 21 \\
Female & \\
Location of the lesions & $8(17.4 \%)$ \\
Neck & $25(54.3 \%)$ \\
Abdominal wall & $13(28.3 \%)$ \\
Axilla/lateral chest wall & \\
Size of the lesions, cm & $18 \mathrm{~cm} \times 12 \mathrm{~cm}$ \\
The largest size & $6.3 \mathrm{~cm} \times 6 \mathrm{~cm}$ \\
The smallest size & $10.6 \mathrm{~cm} \times 7.2 \mathrm{~cm}$ \\
Average size & \\
Prior treatment & \\
Surgery & \\
No prior treatment & \\
\hline
\end{tabular}

\section{Evaluation of outcome}

We assessed the final result of sclerotherapy on the basis of a multidisciplinary consensus during the patient's followup visits, considering the objective and subjective results at each visit. The effect of sclerotherapy was assessed by physical examination and MR imaging 3-6 months after the last procedure. The treatment outcome was divided into four grades based on post-procedure imaging findings at least 2 months after the last session of sclerotherapy therapy: excellent, $>80 \%$ decrease in size; moderate, 50 $80 \%$ decrease in size; mild, $10-50 \%$ decrease in size; and no change, $<10 \%$ decrease in size. No further sclerotherapy was recommended by multidisciplinary consensus on cases with signs of no change or deterioration, indicating ineffective treatment. For the patients who received sclerotherapies for more than 6 times, chest $\mathrm{X}$-ray plain film was detected to observe pulmonary conditions 6 months later after the last procedure.

\section{Results}

Twenty-five male and 21 female patients were included in the study. The age was ranged from 6 to 37 years. The microcystic LMs located in the neck $(\mathrm{n}=8,17.4 \%)$, abdominal wall $(\mathrm{n}=25,54.3 \%)$, and axilla/lateral chest wall $(\mathrm{n}=13$, $28.3 \%)$. The largest lesion measured was $18 \mathrm{~cm} \times 12 \mathrm{~cm}$ and the smallest one was $6.3 \mathrm{~cm} \times 6 \mathrm{~cm}$, with an average area of $10.6 \mathrm{~cm} \times 7.2 \mathrm{~cm}$. Twelve patients had history of surgical resection. Six patients had lymphatic fluid leakage which was the main symptom influencing the quality of life. Other complained symptoms including focal swelling and concerns regarding appearance. The demographic and clinical data of all the subjects are summarized in Table 1 .

Bleomycin was the only sclerosant used for microcystic LMs. The average treatment times were 4.5 times (range, 3 to 8 times) with a mean dose of $7.3 \mathrm{mg}$ (range, 5 to $10 \mathrm{mg}$ ) at a single session. The mean follow-up time was 2.8 years (range, 2 months to 5 years). Based on clinical manifestations and MR findings, an excellent outcome was achieved in 32 patients $(69.6 \%)$, a moderate decrease in 11 patients $(23.9 \%)$, a mild outcome in 3 patients $(6.5 \%)$ (Table 2). No cases of deterioration were observed. The lymphatic fluid leakage was effectively cured after 2-3 courses and the quality of life was improved dramatically. There were 6 patients (13\%) with a long-term follow-up time over 3 years after the last therapeutic session, and no recurrence was examined by MRL.

One of the most commonly occurred adverse events was evident postoperative localized swelling, which subsided in 3 to 5 days, and showed in 7 patients (15.2\%). We listed it as one of adverse events because most of the patients after sclerotherapy showed very mild swelling that could be ignored. The other one was hyperpigmentation in the injection area and was closely associated with the number of injections ( $\mathrm{n}=10,21.7 \%)$. There were 3 patients $(6.5 \%)$ with slight intralesional hemorrhage, and 5 patients (10.9\%) with low-grade fever, both of them resolved spontaneously. No pain, infection, reactive erythema, allergy, and leakage from puncture sites were observed in all the patients. For the 4 patients $(8.7 \%)$ who received sclerosing treatment for more than 6 times and were followed up for more than 5 years, chest $\mathrm{X}$-ray radiograph pre- and post-procedure were compared, and no lung fibrosis occurred (Table 2).

\section{Case 1}

A 22-year-old man suffered from microcystic LMs since birth, and received the first surgical resection when he was 5 years old. The lesion recurred and aggravated gradually, then he received reoperation at 17 years of age followed with evident recurrence 5 years later. The pathological diagnosis from a biopsy performed at another hospital was 
Table 2 Treatment outcomes and adverse effects

\begin{tabular}{lc}
\hline Outcomes & Number (\%) \\
\hline Treatment times & $3-8$ \\
Range & 4.5 \\
Average & \\
Treatment effect & $32(69.6)$ \\
Excellent & $11(23.9)$ \\
Moderate & $3(6.5)$ \\
Mild & \\
Adverse effect & $7(15.2)$ \\
Localized swelling & $10(21.7)$ \\
Hyperpigmentation & $3(6.5)$ \\
Slight hemorrhage & $5(10.9)$ \\
Low-grade fever & 0 \\
Pain & 0 \\
Infection & 0 \\
Erythema & 0 \\
Allergy & 0 \\
Pulmonary fibrosis & \\
\hline
\end{tabular}

lymphangioma. A physical examination showed multiple microcysts, which were prominent at the left lower back, and the involved area was approximately $15 \mathrm{~cm} \times 10 \mathrm{~cm}$ with local swelling (Figure 2A). The lower region of the lesion had two overlying surgical scars. MRI showed that microcystic spaces were confined to the subcutaneous tissue and did not penetrate into the deep fascia and presented as high signal intensity on spin echo $\mathrm{T} 2$ sequences with fat suppression (Figure 2B,C). The lesion area was marked according to ultrasound and MRI detection, bleomycin sclerotherapy of 3 sessions with an interval of 4 weeks was performed as previously described. The area contained no evident cysts but post-inflammatory pigmentation (Figure 2D). Postoperative MRI showed no dilated cysts or channels but fibrosis scar tissue in the lesion area in the MRI images 3 years after the last treatment session, indicating there was no recurrence (Figure 2E,F).

\section{Case 2}

A male patient aged 28 years had a right axillary lesion since birth. He received surgical resection and skin grafting over the defect at 18 and 25 years old, respectively. A physical examination showed multiple microcysts in the flexor side of the right arm that overlapped with the skin graft and scar tissue, and extended to the right chest wall (Figure $3 A$ ). Ultrasound showed two microcystic areas of $4.9 \times 3.8 \mathrm{~cm}$ and $7.5 \mathrm{~cm} \times 3.6 \mathrm{~cm}$ infiltrating into the muscular layer, which was demonstrated by pretreatment MRL (Figure 3B,C). Thickening and hyperintensity of the cephalic vein along its course through the cystic lesion were observed (Figure 3B). Bleomycin sclerotherapies of 5 sessions were performed at a 4- to 6-week-interval with a total dose of $10 \mathrm{mg}$ each time. The patient achieved an excellent outcome after the therapy. There was no recurrence after a 5 -year follow-up evaluated by MRL (Figure 3D,E,F).

\section{Discussion}

Microcystic LMs are congenital benign cutaneous lesions that are characterized by aggregation of low-flow microscopic lymphatic channels, mainly affect skin and mucosa (17). LMs grow proportionally with the child. It is always taken into attention when a lesion-related complication appears, such as swelling, infection, or spontaneous hemorrhage. The treatment indications for LMs include recurrent swelling, pain, infections, functional impairment, and leakage of fluid from the lesions into body cavities or skin.

The microcystic lesions consist of tiny cavities that produce a hyperechoic, solid appearance and can be diagnosed by ultrasound and MRI. Ultrasound could detail the extent, components, and size of the lesion. MRI could detect extent and relation with other anatomical structures and is particularly useful for malformations that involve muscle as T2-weighted scan can distinguish them from normal muscle (18). In recent years, we used MRL as a routine detection for evaluating lymphatic morphology and function in disorders of the lymphatic circulation because it could provide good positioning and three-dimensional (3D) imaging for more accurate treatment and follow-up than other techniques $(11,19)$.

Sclerotherapy has been demonstrated as an effective and less invasive method for LMs, and satisfactory results have been obtained with various sclerosants with certain curative results and some limitations and side effects for each of these agents, including OK-432 (Picibanil), doxycycline, absolute alcohol (99.5\% ethanol), sodium morrhuate, sirolimus and bleomycin. It has been reported that OK432 injection could provide a regression of lesions in up to 

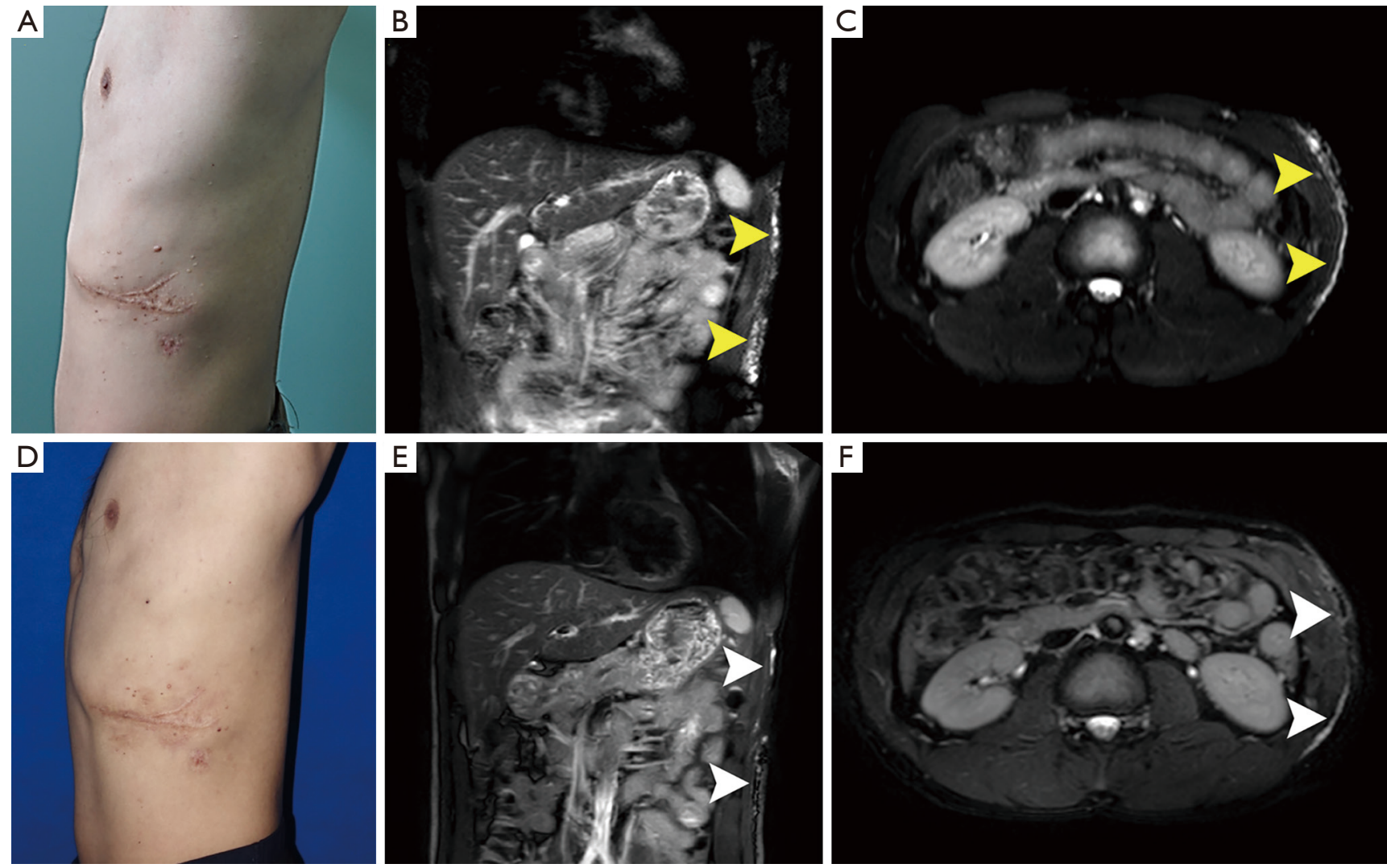

Figure 2 Bleomycin sclerotherapy for recurrent microcystic lymphatic malformations (LMs). (A) Pretherapeutic view of the skin in the abdominal wall, presenting multiple tiny cysts scattered in an area of $15 \mathrm{~cm} \times 10 \mathrm{~cm}$ around the two surgical scars. (B) Before sclerotherapy, coronal T2-weighed (T2W1)/turbo spin echo (TSE) magnetic resonance image (MRI) demonstrated tiny cysts located in the subcutaneous tissue and did not penetrate into the deep fascia. The cysts presented as high-signal intensity because of containing lymphatic fluid (yellow arrow heads). (C) On axial T2W1/TSE MRI, the hyper-intensity of the lesions was apparent before treatment (yellow arrow heads). (D) The area contained no evident cysts but post-inflammatory pigmentation. (E,F) The MRI images showed that the cysts have disappeared, replaced by fibrosis scar tissue in the area (white arrow heads).

$96 \%$ of patients (20). However, this agent is not currently available in China. As for doxycycline, it always causes severe discomfort on injection, requiring general anesthesia in most patients, and the pain within 1 to 3 hours after injection was common and requires narcotic analgesic. In addition, high overall dose is always needed to achieve good results, following with high complications rates, especially in children (9). Absolute alcohol has powerful sclerosing properties, inducing sloughing of the endothelium and obliteration of the lumen, but the possible side effects are much higher than other agents, such as nerve injury, skin necrosis, and hypotension (21). Sodium morrhuate has also been used in LMs, it is widely available but not commonly used because of significant side effects, including anaphylactic reactions, skin necrosis, and pain (22).
Microcystic LMs are less responsive to conventional percutaneous sclerotherapy techniques because of the smaller dimensions of the cysts or channels than macrocystic LMs (20). However, they own same pathologic features. Therefore, based on the appropriate injection way, it is achievable to infuse enough concentration and dose of sclerosants in microcystic LMs to obtain efficient tissue inflammation and therapy outcomes. Among all these sclerosants, bleomycin sclerotherapy is mostly used in microcystic LMs. Good results in efficacy and safety have been obtained by bleomycin injection, demonstrated by $40-60 \%$ complete resolution and 30\% remarkable reduction in size $(23,24)$. Based on its efficacy and easy accessibility, we used bleomycin sclerotherapy as a first-line management on microcystic LMs in our unit. 

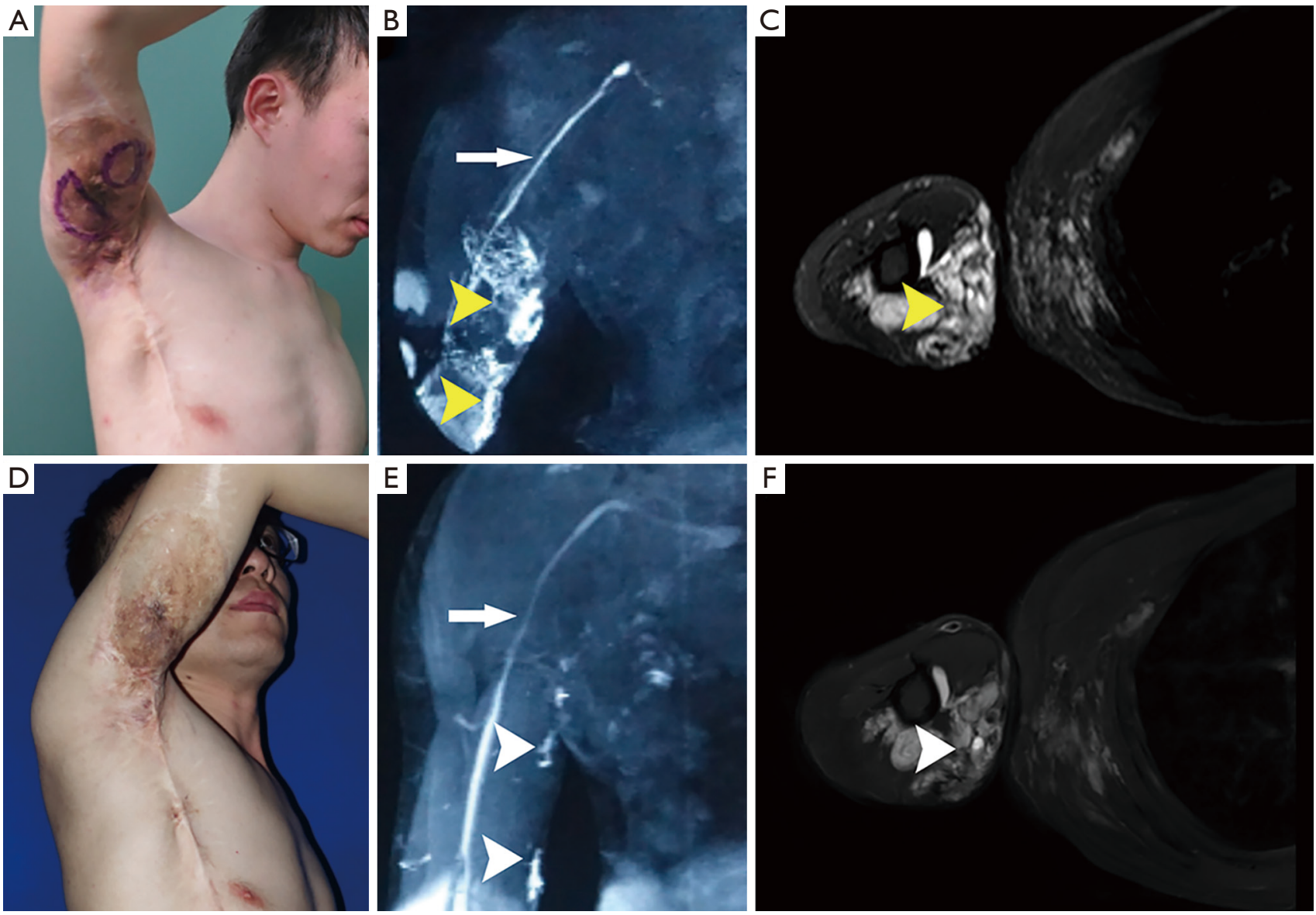

Figure 3 Axillary microcystic lymphatic malformations (LMs) before and after treatment. 3D spoiled gradient-recalled T1-weighted highresolution isotropic volume examination sequence and 3D maximum intensity projection imaging technique were performed to reconstruct the images. (A) Microcystic LMs in the right axillary area before sclerotherapy. The proximal arm was swollen and the scope of the lesion was located by ultrasound detection. (B) The pre-therapeutic image clearly showed overlapped lymph cysts, which infiltrated into the muscle (yellow arrow heads). The grey arrow indicates the cephalic vein (white arrow). (C) On axial T2W1/TSE MRI, the hyper-intensity of the lesions was apparent before treatment and it clearly showed the lesion infiltrated the muscle (yellow arrow heads). (D) Five years after treatment, there was no remaining skin lesion but hyperpigmentation. (E,F) Five years after bleomycin sclerotherapies of 5 sessions, the corresponding hyperintense area in the axillary area disappeared, replaced by fibrosis scar tissue in the area (white arrow heads).

Until now, few published studies have evaluated the objective response in the treatment of bleomycin on large diffuse microcystic LMs. Our study, for the first time, demonstrated that the combined methods of local lavage and intradermal injection were effective and safe to treat large diffuse microcystic LMs with low risk of complications and negligible lung toxicity. The success rate was $92.4 \%$ (excellent and moderate results), which was similar with the previous reports about bleomycin sclerotherapy in microcystic LMs $(14,15)$.

The most common complications postoperatively are evident local swelling, slight intralesional hemorrhage, low-grade fever, which would subside within 2-3 days. Pigmentation in the injection area was difficult to resolve spontaneously and can be improved by laser treatment. Pulmonary fibrosis by bleomycin injection is a major concern. However, until now, it has reached a consensus that bleomycin is a safe sclerosant because there were no complications when no more than $15 \mathrm{U}$ in adults or $0.5 \mathrm{U} / \mathrm{kg}$ per session in children and a cumulative dose $<90 \mathrm{U}$ in adults or 6 sessions of $0.5 \mathrm{U} / \mathrm{kg}$ in children were administrated $(14,24)$. To reduce the potential risk, previous ultrasound guidance can be performed to increase the accuracy. Furthermore, intralesional bleomycin lavage 
in larger cysts could minimize drug absorption, and the dose of bleomycin should be strictly controlled. For the patients who received more than 6 sessions in our study, there was no pulmonary fibrosis detected with chest X-ray radiograph. However, there was no quantitative data about lavage volume in each cyst in our study, which will be calculated and documented in our further work to explore precise therapy. The other complication that should be paid attention to was hyperpigmentation, laser treatment is effective to decrease it. In cases of superficial lesions of the face and neck with risk of subsequent poor cosmetic result, a dilution solution can be utilized (25).

On review of the literature, there were reports about bleomycin toxicity and hypersensitivity. In Niramis's study, leukopenia was noted in 3 patients below one year of age, which might be caused by bleomycin toxicity, and the author suggested the dosage of bleomycin should be reduced in infants (26). Bleomycin hypersensitivity is a rare emergency. Until now, there was one reported case of pulmonary toxicity following $1.2 \mathrm{mg} / \mathrm{kg}$ single session dose in an 8-month-old infant, and received corticosteroid, pentoxifylline and supportive care to relieve symptoms (27). Therefore, it is essential to avoid injecting the drug into blood vessels and give close observation in the first 24 hours, especially in infants.

Bleomycin sclerotherapy has a number of potential advantages over excision, including little damage to local structures, low recurrence, short hospital stays. In a retrospective cohort study about head and neck LMs, sclerotherapy has demonstrated similar outcomes to primary surgery after 1 year, without differences in resource utilization (28). In addition, sclerosing treatment is not affected by the location and size of the lesion. Although multiple treatments may be required for large microcystic LMs, most of the patients regard repeated sclerotherapy as a more acceptable choice for total suffering and risk, both of which are much lower than surgical treatment. In other words, sclerotherapy could be considered as the initial treatment modality for these lesions, and surgery could be reserved for cases of sclerotherapy failure or when urgent intervention is needed.

\section{Conclusions}

Good efficacy and low complication rates of bleomycin sclerotherapy in large diffuse microcystic LMs were obtained with combined methods of local lavage and intradermal injection either for the initial treatment or any recurrences after surgery. It is minimally invasive with a low recurrence, and can be regarded as the mainstay of therapy for microcystic LMs to avoid or decrease surgery trauma. To reduce the potential risk of pulmonary fibrosis, bleomycin lavage is recommended to minimize the drug absorption.

\section{Acknowledgments}

We thank Tanja Herrler (Plastic and Reconstructive Surgery, Trauma Center Murnau, Murnau, Germany) for the language editing.

Funding: This work was supported by Shanghai Municipal Key Clinical Specialty (shslczdzk00901).

\section{Footnote}

Reporting Checklist: The authors have completed the STROBE reporting checklist. Available at https://dx.doi. org/10.21037/gs-21-70

Data Sharing Statement: Available at https://dx.doi. org/10.21037/gs-21-70

Peer Review File: Available at https://dx.doi.org/10.21037/ gs-21-70

Conflicts of Interest: All authors have completed the ICMJE uniform disclosure form (available at https://dx.doi. org/10.21037/gs-21-70). The authors have no other conflicts of interest to declare.

Etbical Statement: The authors are accountable for all aspects of the work in ensuring that questions related to the accuracy or integrity of any part of the work are appropriately investigated and resolved. All procedures were performed in accordance with the ethical standards of the institutional review board (Approval from Comissão de Ética do Hospital dos SAMS, 002/2018) and with the 1964 Helsinki declaration and its later amendments (as revised in 2013) or comparable ethical standards. This study was approved by the research and ethics committee of the Shanghai Ninth People's Hospital (Registration number: 2019042). Written informed consent was obtained from all patients.

Open Access Statement: This is an Open Access article distributed in accordance with the Creative Commons 
Attribution-NonCommercial-NoDerivs 4.0 International License (CC BY-NC-ND 4.0), which permits the noncommercial replication and distribution of the article with the strict proviso that no changes or edits are made and the original work is properly cited (including links to both the formal publication through the relevant DOI and the license). See: https://creativecommons.org/licenses/by-nc-nd/4.0/.

\section{References}

1. Yang Y, Sun M, Ma Q, et al. Bleomycin A5 sclerotherapy for cervicofacial lymphatic malformations. J Vasc Surg 2011;53:150-5.

2. Hochman M, Adams DM, Reeves TD. Current knowledge and management of vascular anomalies, II: malformations. Arch Facial Plast Surg 2011;13:425-33.

3. Elluru RG, Balakrishnan K, Padua HM. Lymphatic malformations: diagnosis and management. Semin Pediatr Surg 2014;23:178-85.

4. Luzzatto C, Midrio P, Tchaprassian Z, et al. Sclerosing treatment of lymphangiomas with OK-432. Arch Dis Child 2000;82:316-8.

5. Ayhan E. Lymphangioma Circumscriptum: Good Clinical Response to Isotretinoin Therapy. Pediatr Dermatol 2016;33:e208-9.

6. Ba aran MM, Karatayli SO, Pampal A, et al. Successful Treatment of a Case with Cervical Lymphatic Malformation: Repeated Bleomycin Sclerotherapy. Turk Arch Otorhinolaryngol 2017;55:180-3.

7. Horbach SER, Wolkerstorfer A, de Bruin DM, et al. Electrosclerotherapy for capillary malformations: study protocol for a randomised within-patient controlled pilot trial. BMJ Open 2017;7:e016401.

8. Schulz D, Lein A, Nicula AP, et al. Lymphangioma Circumscriptum Post Radiotherapy for Penile Cancer Treated with $\mathrm{CO}(2)$ Laser. Acta Dermatovenerol Croat 2018;26:53-7.

9. Burrows PE, Mitri RK, Alomari A, et al. Percutaneous sclerotherapy of lymphatic malformations with doxycycline. Lymphat Res Biol 2008;6:209-16.

10. Nehra D, Jacobson L, Barnes P, et al. Doxycycline sclerotherapy as primary treatment of head and neck lymphatic malformations in children. J Pediatr Surg 2008;43:451-60.

11. Jiang Z, Li S, Kretlow JD, et al. Closure of large defects after microcystic lymphatic malformations using lateral intercostal artery perforator flap. J Plast Reconstr Aesthet Surg 2014;67:1230-6.
12. Bagrodia N, Defnet AM, Kandel JJ. Management of lymphatic malformations in children. Curr Opin Pediatr 2015;27:356-63.

13. Wang H, Li S, Jiang Z. Magnetic resonance lymphangiography for the assessment of the lymphatic system in a lymphatic malformation patient undergoing sclerotherapy. J Dermatol 2016;43:981-3.

14. Chaudry G, Guevara CJ, Rialon KL, et al. Safety and efficacy of bleomycin sclerotherapy for microcystic lymphatic malformation. Cardiovasc Intervent Radiol 2014;37:1476-81.

15. Da Ros V, Iacobucci M, Puccinelli F, et al. Lymphographic-Like Technique for the Treatment of Microcystic Lymphatic Malformation Components of $<3$ mm. AJNR Am J Neuroradiol 2018;39:350-4.

16. Yura J, Hashimoto T, Tsuruga N, Shibata K. Bleomycin treatment for cystic hygroma in children. Nihon Geka Hokan 1977;46:607-14.

17. Hogeling M, Adams S, Law J, et al. Lymphatic malformations: clinical course and management in 64 cases. Australas J Dermatol 2011;52:186-90.

18. Chisin R, Fabian R, Weber AL, et al. MR imaging of a lymphangioma involving the masseter muscle. J Comput Assist Tomogr 1988;12:690-2.

19. Borri M, Schmidt MA, Gordon KD, et al. Quantitative Contrast-Enhanced Magnetic Resonance Lymphangiography of the Upper Limbs in Breast Cancer Related Lymphedema: An Exploratory Study. Lymphat Res Biol 2015;13:100-6.

20. Giguère CM, Bauman NM, Sato Y, et al. Treatment of lymphangiomas with OK-432 (Picibanil) sclerotherapy: a prospective multi-institutional trial. Arch Otolaryngol Head Neck Surg 2002;128:1137-44.

21. Nassiri N, Rootman J, Rootman DB, Goldberg RA. Orbital lymphaticovenous malformations: Current and future treatments. Surv Ophthalmol 2015;60:383-405.

22. Schwarcz RM, Ben Simon GJ, Cook T, Goldberg RA. Sclerosing therapy as first line treatment for low flow vascular lesions of the orbit. Am J Ophthalmol 2006;141:333-9.

23. Okada A, Kubota A, Fukuzawa M, et al. Injection of bleomycin as a primary therapy of cystic lymphangioma. J Pediatr Surg 1992;27:440-3.

24. Sainsbury DC, Kessell G, Fall AJ, et al. Intralesional bleomycin injection treatment for vascular birthmarks: a 5 -year experience at a single United Kingdom unit. Plast Reconstr Surg 2011;127:2031-44.

25. Muir T, Kirsten M, Fourie P, et al. Intralesional bleomycin 
injection (IBI) treatment for haemangiomas and congenital vascular malformations. Pediatr Surg Int 2004;19:766-73.

26. Niramis R, Watanatittan S, Rattanasuwan T. Treatment of cystic hygroma by intralesional bleomycin injection: experience in 70 patients. Eur J Pediatr Surg 2010;20:178-82.

27. Atwa K, Abuhasna S, Shihab Z, et al. Acute pulmonary

Cite this article as: Sheng L, Yu Z, Li S, Cao W, Jiang Z. Bleomycin sclerotherapy for large diffuse microcystic lymphatic malformations. Gland Surg 2021;10(6):1865-1873. doi: 10.21037/gs$21-70$ toxicity following intralesional administration of bleomycin for a lymphovenous malformation. Pediatr Pulmonol 2010;45:192-6.

28. Balakrishnan K, Menezes MD, Chen BS, et al. Primary surgery vs primary sclerotherapy for head and neck lymphatic malformations. JAMA Otolaryngol Head Neck Surg 2014;140:41-5. 